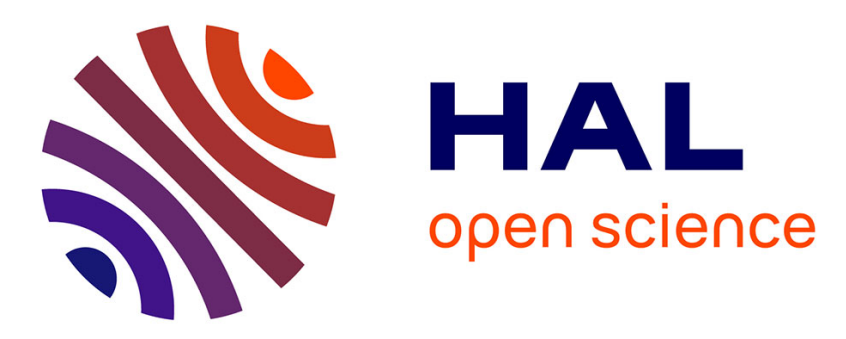

\title{
Production de plasmas denses par explosion de filament gazeux ionisé
}

M. Skowronek, P. Romeas, Vu-Thien-Gia

\section{To cite this version:}

M. Skowronek, P. Romeas, Vu-Thien-Gia. Production de plasmas denses par explosion de filament gazeux ionisé. Revue de Physique Appliquée, 1977, 12 (10), pp.1723-1727. 10.1051/rphysap:0197700120100172300 . jpa-00244393

\section{HAL Id: jpa-00244393 https://hal.science/jpa-00244393}

Submitted on 1 Jan 1977

HAL is a multi-disciplinary open access archive for the deposit and dissemination of scientific research documents, whether they are published or not. The documents may come from teaching and research institutions in France or abroad, or from public or private research centers.
L'archive ouverte pluridisciplinaire HAL, est destinée au dépôt et à la diffusion de documents scientifiques de niveau recherche, publiés ou non, émanant des établissements d'enseignement et de recherche français ou étrangers, des laboratoires publics ou privés. 
Classification

Physics Abstracts

$52.00-52.50 \mathrm{~L}$

\title{
PRODUCTION DE PLASMAS DENSES PAR EXPLOSION DE FILAMENT GAZEUX IONISÉ
}

\author{
M. SKOWRONEK, P. ROMEAS et VU-THIEN-GIA
}

Laboratoire des Plasmas Denses, L.P.O.C., Université Pierre-et-Marie-Curie, T. 12, E 5, 75230 Paris Cedex 05, France

(Reçu le 22 décembre 1976, révisé le 31 mai 1977, accepté le 27 juin 1977)

\begin{abstract}
Résumé. - Une méthode de production de plasma dense par explosion de filament gazeux ionisé est décrite. Les résultats des mesures électriques et spectrophotométriques montrent l'efficacité d'une injection rapide de l'énergie dans la colonne de plasma, pour deux expériences de caractéristiques temporelles très différentes.
\end{abstract}

\begin{abstract}
A method for producing a dense plasma by explosion of a thin preionized gaseous filament is described. The electric and spectrophotometric results show the efficiency of a rapid injection of the energy in the plasma column in two experiments which have different durations.
\end{abstract}

1. Introduction. - L'étude théorique des plasmas denses a considérablement progressé ces dernières années. Les diverses méthodes employées : simulation numérique des plasmas à une composante [1], étude du gaz coulombien à deux dimensions [2], thermodynamique statistique [3] permettent le calcul des fonctions thermodynamiques, des états d'ionisation et des coefficients de transport, des plasmas denses à forte corrélation pour lesquels l'énergie d'interaction coulombienne est du même ordre que l'énergie cinétique.

Parallèlement, les expérimentateurs ont déployé une grande activité pour produire des plasmas denses et chauds dans la perspective d'applications telles que : générateurs M.H.D., propulsion spatiale, fusion thermonucléaire contrôlée, où l'on prévoit que l'état de plasma dense constituera le fluide de travail. Dans les machines du type Focus [4], le plasma est chauffé électriquement et comprimé magnétiquement dans une configuration non symétrique. Dans les plasmas créés par laser, le rayonnement est emprisonné et le confinement est inertiel [5]. Dans d'autres cas, on utilise l'énergie électrique pour mettre en mouvement implosif un cylindre et créer ainsi un plasma dense thermonucléaire [6]. La méthode du fil explosé [7] produit un plasma difficile à utiliser à cause des problèmes liés à la fusion et à la volatilisation du fil.

Dès 1954, Allen et Craggs utilisaient une étincelle intense pour produire un plasma de $N_{\mathrm{e}} \simeq$ $3 \times 10^{19} \mathrm{~cm}^{-3}$ et $T_{\mathrm{e}} \simeq 10 \mathrm{eV}$; le courant atteignait $265 \mathrm{kA}$ en $7,7 \mu \mathrm{s}$ [10]. En 1967, Borovick et al. [11] utilisent des fils explosés sous haute pression (jusqu'à 1000 bars) pour créer des plasmas jusqu'à $10^{20} \mathrm{~cm}^{-3}$.

La méthode, que nous employons, consiste à créer une colonne de gaz ionisé de faible diamètre dans laquelle nous déchargeons l'énergie stockée dans une batterie de condensateurs [8]. Nous échauffons par effet Joule le plus petit volume de plasma possible par utilisation de l'inertie du gaz qui entoure le plasma et qui s'oppose à son expansion. La décharge se développe cylindriquement à partir du canal initial et sa localisation spatiale est très précise, ce qui permet une analyse correcte d'une petite portion de la décharge. La matière se rassemble à la périphérie de la décharge, formant une couche très dense et opaque qui emprisonne le rayonnement du cœur de la décharge. Cette circonstance favorise la surchauffe du plasma [9]. La situation est bien différente pour les claquages laser où l'augmentation de la densité électronique et l'arrivée de la fréquence de plasma au voisinage de la fréquence du laser provoque la réflexion de l'énergie lumineuse.

Nous décrivons dans la suite, le dispositif expérimental, les résultats des mesures spectrophotométriques effectuées sur la gaine externe, les résultats des mesures électriques qui intéressent le cœur de la décharge et nous discutons le mécanisme de la conduction.

2. Dispositif expérimental. - Un filament stable de gaz ionisé, de faible diamètre, de l'ordre de 10 à $20 \mu \mathrm{m}$ est établi dans l'air atmosphérique, par transition 
couronne-arc, entre pointes fines [12]. Dans ce filament, où passe un courant de $10 \mu \mathrm{A}$, un système d'éclateurs permet, à un instant donné, de décharger l'énergie contenue dans un banc de condensateurs dont la capacité est $2,6 \mu \mathrm{F}$ et dont la tension de charge peut atteindre $100 \mathrm{kV}$. Les expériences décrites ici ont été réalisées à $80 \mathrm{kV}$ avec une énergie stockée de $8 \mathrm{~kJ}$. Chacun des condensateurs est muni d'un éclateur coaxial. Ces éclateurs fonctionnent sous pression de $\mathrm{SF}_{6}$ grâce à une impulsion de $60 \mathrm{kV}$ dont le temps de montée est inférieur à 100 ns. L'incertitude sur le déclenchement est inférieur à 20 ns [13]. L'énergie électrique est amenée par des câbles coaxiaux à la chambre d'expériences, également de géométrie coaxiale. La pseudo-période du courant de décharge est ramenée à $3,8 \mu$ s ce qui correspond à une inductance totale du circuit de $140 \mathrm{nH}$.

Le circuit de décharge est équivalent à un condensateur $C$, une inductance $L$ et une résistance $R$ montés en série dont deux éléments ( $L$ et $R$ ) sont rapidement variables. C'est ce qui explique pourquoi la première arche de courant s'écarte de la sinudoïde traditionnelle [14]. La mesure du courant de décharge est faite au moyen d'une bobine de Rogowski dont le temps de montée est $10 \mathrm{~ns}$.

Une caméra à miroir tournant enregistre l'expansion de la décharge. La vitesse d'expansion du front de décharge est environ de $4 \mathrm{~km} / \mathrm{s}$. Accompagnant ce front, il est possible de mettre en évidence une onde de choc intense se propageant à Mach 12 dans l'air à la pression atmosphérique. L'expansion de la décharge se fait à vitesse constante pendant 3 ou $4 \mu \mathrm{s}$.

L'image de la décharge agrandie dix fois est projetée sur la fente d'un monochromateur muni d'un photomultiplicateur. Nous mesurons ainsi la luminance d'une portion très réduite de l'aire de la décharge. Seule, une localisation précise de la décharge nous permet d'effectuer cette mesure et d'obtenir des signaux lumineux dont les temps de montée soient très brefs : de 300 à 500 ns suivant la longueur d'onde.

La chaîne de mesure des luminances est soigneusement étalonnée par rapport à une lampe à ruban de tungstène et à l'arc au carbone. Dans l'infrarouge à $2,2 \mu$ et $4,8 \mu$ l'intensité de la lumière est mesurée après passage dans un filtre interférentiel à l'aide d'une cellule rapide refroidie à l'azote liquide.

3. Résultats des mesures de luminance spectrale. En projetant la lumière d'un laser à rubis sur la décharge, nous avions constaté que celle-ci était opaque dès la première centaine de nanosecondes [8]. Nous avons mesuré la température de luminance, à chaque instant de la décharge pour un grand nombre de longueurs d'onde. Si la décharge était un corps noir, cette température devrait être constante suivant les longueurs d'onde. Les variations que nous observons sont attribuées à des variations de l'émissivité spectrale de plasma : $\varepsilon_{\lambda}=\frac{B_{\lambda}}{B_{0}}$.

$B_{\lambda}$ : Luminance à longueur d'onde $\lambda$ de la décharge. $B_{0 \lambda}$ : Luminance du corps noir à la même longueur d'onde et à la même température. Cette température est évaluée d'après la courbe des températures de luminance.

L'émissivité d'une couche homogène plane, d'épaisseur $x$, caractérisée par les coefficients de réflexion $R$ et d'absorption $K$ est donnée par :

$$
\varepsilon=\frac{B}{B_{\mathrm{o}}}=(1-R) \frac{1-\exp -K x}{1-R \exp -K x}
$$

$K$ et $R$ sont calculés à chaque longueur d'onde en introduisant des valeurs de la fréquence de collisions $f_{\mathrm{C}}$ et de la fréquence de plasma $f_{\mathrm{p}}$. Le paramètre $x$ permet de calculer ensuite $\varepsilon$. On cherche, en modifiant les quantités $f_{\mathrm{C}}, f_{\mathrm{p}}$ et $x$ à ajuster $(\varepsilon \lambda)$ calculé à $(\varepsilon \lambda)_{\exp }$. Les remarques suivantes accélèrent les approximations : la fréquence de plasma $f_{\mathrm{p}}$ intervient surtout pour situer la chute de luminance au passage de cette fréquence vers l'infrarouge; la fréquence de collisions $f_{\mathrm{C}}$ intervient surtout pour le coefficient de réflexion; l'épaisseur de la couche de plasma intervient dans la baisse de luminance vers l'ultra-violet. L'ajustement nous fournit donc l'ensemble des paramètres intéressant la décharge. Nous avons reporté sur le tableau I les valeurs caractérisant le plasma de la couche superficielle pour deux types de décharge de périodes différentes : l'une à $10 \mu \mathrm{s}$ [8] et l'autre à $3,8 \mu$ s.

Le plasma de la gaine est donc un plasma très dense, à forte corrélation. Le nombre de particules dans la sphère de Debye est inférieur à l'unité. Ce plasma présente un intérêt certain pour l'étude de son interaction avec le rayonnement laser en liaison avec les recherches sur l'implosion laser.

Le schéma simplifié qu'on peut présenter de la décharge est le suivant :

a) la décharge présente une gaine très dense de faible épaisseur, dont l'analyse est faite optiquement.

TABLEAU I

$\begin{array}{lcccccc} & \begin{array}{c}\text { Luminance } \\ \text { max. ̀̀ }\end{array} & T_{\mathrm{e}} & N_{\mathrm{e}} & \text { Épaisseur } & f_{\mathrm{C}} / f_{\mathrm{P}} & \text { Pression } \\ \begin{array}{l}\text { Décharge } \\ \text { lente } T=10 \mu \mathrm{s}\end{array} & \boldsymbol{\tau}=0,6 \mu \mathrm{s} & -4 \mathrm{eV} & 10^{21} \mathrm{~cm}^{-3} & x=20 \mu \mathrm{m} & 8 \times 10^{-2} & 5,5 \mathrm{kbar} \\ \begin{array}{l}\text { Décharge } \\ \text { rapide } T=3,8 \mu \mathrm{s}\end{array} & \boldsymbol{\tau}=0,4 \mu \mathrm{s} & 10 \mathrm{eV} & 10^{21} \mathrm{~cm}^{-3} & x=5 \mu \mathrm{m} & 2 \times 10^{-2} & 14 \mathrm{kbar}\end{array}$


b) le cœur chaud et moins dense influence les caractéristiques électriques. Nous allons le décrire maintenant.

4. Caractéristique de la zone axiale déduite des mesures électriques. - La décharge des condensateurs s'effectue au travers d'une résistance variable $R$.

$R$ est une somme de plusieurs termes qu'on peut écrire $R=R_{\mathrm{o}}+R_{1}+R_{2}$.

$R_{\mathrm{o}}$ : résistance des câbles. Elle est constante et très faible.

$R_{1}$ : résistance des éclateurs. $R_{1}<10^{-2} \Omega$.

$R_{2}$ : résistance de la décharge. Pendant la première demi période $R_{2}>R_{\mathrm{o}}+R_{1}$ et on peut confondre $R_{2}$ avec $R$.

La résistance résiduelle $R_{\mathrm{o}}+R_{1}$ peut être appréciée d'après le décrément logarithmique au bout d'un temps assez long lorsque $R_{2}$ devient négligeable.

La loi d'Ohm pour le circuit s'écrit :

$$
V_{0}=\frac{1}{c} \int_{0}^{t} i \mathrm{~d} t+R i+L \frac{\mathrm{d} i}{\mathrm{~d} t} \text { où } V_{0} \text { est la tension de }
$$

charge des condensateurs. Nous avons négligé le terme $i \frac{\mathrm{d} L}{\mathrm{~d} t}$ qui reste encore petit, même aux fréquences où nous travaillons.

Nous résolvons numériquement cette équation et calculons $R(t)$, résistance de la décharge, rapidement variable avec le temps.

Le terme $R i$ est obtenu par différence; il ne sera connu avec une bonne précision que dans un domaine limité des valeurs de $R i$ : pour les faibles valeurs de $R i: R i>V_{\mathrm{o}} / 5$ et pour les grandes valeurs de $R i$, par suite de l'imprécision sur $i, R$ est mal connu.

4. 1. DÉTERMination DE LA TEMPÉRATURE. Connaissant à chaque instant le diamètre de la décharge, la résistance et le courant, la densité d'énergie électrique déposée dans la décharge s'en déduit. $\mathrm{Si}$ l'on peut évaluer la densité à l'intérieur de la décharge, la température du cour de la décharge est connue.

La densité initiale des particules est $2,7 \times 10^{19} \mathrm{~cm}^{-3}$. Nous avons vu qu'une grande partie de la matière se rassemble en une couche dense à la périphérie. Cependant, il est difficile d'admettre qu'un vide important existe sur l'axe. Il est raisonnable de supposer que la densité sur l'axe soit de l'ordre de $10^{19} \mathrm{~cm}^{-3}$ (compte tenu des ionisations multiples également). La température atteint donc
$1 \mathrm{keV}$ lorsque la densité d'énergie est $1 \mathrm{~kJ} / \mathrm{cm}^{3}$. Le même résultat est obtenu lorsqu'on écrit l'égalité des pressions au cœur et sur la gaine.

Le tableau II ci-dessous donne les paramètres principaux au cœur des deux décharges que nous avons déjà comparés.

L'efficacité d'une injection rapide de l'énergie est donc bien mise en évidence.

4. 2. Application de la théorie de WeizelROMPE [15]. - L'équation reliant les divers modes de production et de déperdition d'énergie s'écrit :

$$
\rho \frac{\mathrm{D} u}{\mathrm{D} t}=\frac{j^{2}}{\sigma}-p \operatorname{div} v-\operatorname{div} Q
$$

Les différents symboles ont la signification usuelle :

$\rho:$ densité du milieu,

$u$ : densité d'énergie,

$j:$ densité du courant,

$\sigma:$ conductivité,

$p:$ pression,

$v$ : vitesse,

$Q:$ représente les pertes par rayonnement et condition thermique.

Dans la théorie de Weizel-Rompe, les simplifications suivantes sont faites : les pertes par rayonnement et conduction sont négligées ainsi que le travail de la pression. On suppose une température homogène et une relation linéaire entre la conductivité et la température: $\sigma=a T$.

L'énergie interne est donnée par la formule :

$$
u=\frac{1}{\gamma-1} \frac{p}{\rho}=\frac{2 n k T}{(\gamma-1) \rho}
$$

La formule de Weizel-Rompe s'en déduit :

$$
R(t)=B \frac{l}{\left(\int_{0}^{t} i^{2} \mathrm{~d} t\right)^{1 / 2}}
$$

$l$ est la longueur de la décharge et $B$ est une constante.

En prenant une relation entre la conductivité et la température du type $\sigma \propto T^{3 / 2}$ la relation précédente

\begin{tabular}{|c|c|c|c|c|c|c|c|c|}
\hline & Capacité & $\begin{array}{c}\text { Énergie } \\
\text { stockée } \\
-\end{array}$ & Période & $\underline{I_{\max }}$ & $\begin{array}{c}\text { Temps } \\
\text { pour } \\
I_{\max } \\
-\end{array}$ & $\begin{array}{l}\text { Densité } \\
\text { d'énergie }\end{array}$ & $\underline{T}$ & $\begin{array}{l}\text { Conduc- } \\
\text { tivité }_{\max }\end{array}$ \\
\hline Décharge lente & $4,4 \mu \mathrm{F}$ & $18 \quad \mathrm{~kJ}$ & $10,5 \mu \mathrm{s}$ & $185 \mathrm{kA}$ & $4,3 \mu \mathrm{s}$ & $0,2 \mathrm{~kJ} / \mathrm{cm}^{3}$ & $100 \mathrm{eV}$ & $10(\Omega \mathrm{cm})^{-1}$ \\
\hline Décharge rapide & $2,6 \mu \mathrm{F}$ & $8,35 \mathrm{~kJ}$ & $3,8 \mu \mathrm{s}$ & $305 \mathrm{kA}$ & $1,1 \mu \mathrm{s}$ & $2,5 \mathrm{~kJ} / \mathrm{cm}^{3}$ & $1 \mathrm{keV}$ & $\begin{array}{c}300 \\
(\Omega \mathrm{cm})^{-1}\end{array}$ \\
\hline
\end{tabular}
devient [16].

$$
R=\frac{B l}{\left[\int_{0}^{t} i^{2} \mathrm{~d} t\right]^{0,6}}
$$

TABLEAU II 
Nous avons vérifié cette relation pour la décharge lente et la décharge rapide (voir Fig. 1). Nous trouvons comme exposant, respectivement $0,7 \pm 0,05$ et $0,6 \pm$ 0,05 . Cependant il faut bien reconnaître que la méthode n'est pas sensible à la fonction $\sigma(T)$. Nous avons cherché à calculer la valeur de la constante $B$ afin que la relation soit complète. Nous avons

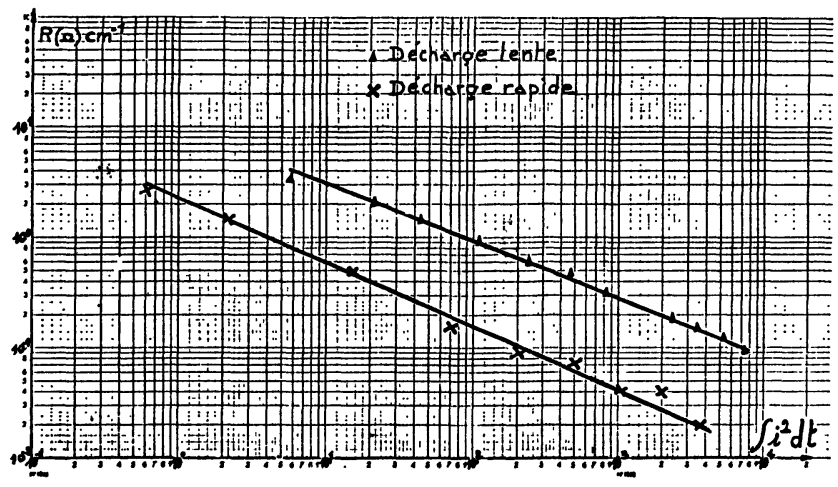

FIG. 1. - Résistance de la décharge par unité de longueur $\left(\Omega \cdot \mathrm{cm}^{-1}\right)$ en fonction de $\int i^{2} \mathrm{~d} t$.

[Resistance of the discharge per unit length $\left(\Omega . \mathrm{cm}^{-1}\right)$ versus $\left.\int i^{2} \mathrm{~d} t.\right]$ trouvé $B=\frac{\bar{P}}{10 \sigma(\gamma-1)}$ où $P$ est une valeur moyenne de la pression sur le temps et l'espace et $\bar{\sigma}$ est une valeur moyenne de la conductivité.

Nous avons constaté que l'accord est bon sur les places respectives des courbes de la figure 1 .

En effet

$\frac{B \text { lente }}{B \text { rapide }}=\frac{P \text { lente }}{P \text { rapide }} \times \frac{\bar{\sigma} \text { rapide }}{\bar{\sigma} \text { lente }}$ or $\frac{\bar{\sigma} \text { rapide }}{\bar{\sigma} \text { lente }}=30$ On admet que $\frac{P \text { lente }}{P \text { rapide }} \simeq \frac{1}{4}$ ce qui permet de calculer $\frac{B \text { lente }}{B \text { rapide }}=7,5$ et le rapport déterminé sur les courbes est environ 6.

On peut expliquer, par l'intervention de la pression magnétique, que l'expansion se fasse à la même vitesse malgré un rapport de pression, quatre fois plus grand.

Nous avions également trouvé un bon accord sur la place des courbes dans le diagramme $R(t)$ fonction de $\left(\int_{0}{ }^{t}{ }^{+2} \mathrm{~d} t\right)$ même pour une décharge dans des conditions totalement différentes, par exemple, les tubes à éclairs [17].

4. 3. MéCAnisme de la CONDUCtion. - Appliquons la formule de Spitzer, au plasma du cœur de la décharge.

$$
\sigma=\frac{1}{3,8 \times 10^{3}} \times \frac{T^{3 / 2}}{Z \log \Lambda} \Omega^{-1} \mathrm{~cm}^{-1}
$$

$Z$ : est la charge des ions. Log $\wedge$ est le logarithme coulombien tabulé dans [18]. Si nous prenons $Z=6$, la formule de Spitzer nous donne la valeur $1,4 \times 10^{5} \Omega^{-1} \mathrm{~cm}^{-1}$ qui est supérieure de plus de deux ordres de grandeurs aux valeurs mesurées qui varient de 300 à $500 \Omega^{-1} \mathrm{~cm}^{-1}$.
La formule de Spitzer n'est applicable qu'aux plasmas en équilibre, où le libre parcours moyen des électrons est beaucoup plus petit qu'une dimension caractérisant le plasma (volume où l'on peut considérer tous les paramètres comme constants). Dans de nombreux cas, les valeurs expérimentales, sont mesurées dans des conditions de turbulence qui rendent la formule de Spitzer inadéquate et ces valeurs sont très inférieures de plusieurs ordres de grandeur. Cette circonstance permet d'ailleurs d'expliquer pourquoi l'on atteint de très hautes températures par chauffage ohmique. Dans les plasmas soumis à des courants à haute fréquence avec des effets de peau importants [19], cette faible conductivité a été mesurée. Dans des plasmas, confinés magnétiquement, qui sont le siège d'instabilités, tels qu'ils furent produits dans les anciens Tokomak [20] cet effet est également reconnu.

Dans le cas de notre décharge, l'hypothèse la plus vraisemblable est que le plasma axial est sujet à des instabilités double-faisceau causées par l'énorme densité de courant qui le parcourt. Les électrons sont animés d'un mouvement d'entraînement par rapport aux ions et excitent des oscillations à la fréquence de plasma. Le critère d'excitation de ces oscillations est une comparaison entre la vitesse thermique des ions et la vitesse d'entraînement des électrons. La vitesse de dérive des électrons est $V_{\mathrm{E}}=\frac{j}{n e}$.

On trouve $V_{E} \simeq 10^{6} \mathrm{~cm} / \mathrm{s}$ alors que la vitesse thermique des ions est de l'ordre $10^{7} \mathrm{~cm} / \mathrm{s}$. Une fraction notable des électrons sera piégée dans les puits de potentiel créés par les oscillations de plasma. Les théoriciens qui ont étudié ce mécanisme proposent, pour calculer la conductivité de remplacer la fréquence de collisions électron-ion par la fréquence de plasma en tant que rythme d'échange d'énergie.

Par exemple, Buneman [21] propose :

$\sigma \simeq \frac{1}{2}\left(\frac{M}{m}\right)^{1 / 3} \omega_{\mathrm{P}}$ où $M$ et $m$ sont les masses respectives des ions et des électrons et $\omega_{\mathrm{P}}$ la fréquence de plasma. Dupree [22] propose la formule $\sigma \simeq 10 \omega_{\mathrm{p}} / k \lambda_{\mathrm{D}}$ où $k$ est un vecteur d'onde moyen et $\lambda_{\mathrm{D}}$ la longueur de Debye. Hamberger [23] a mesuré la conductivité dans des conditions de forte turbulence, à une température de $10 \mathrm{keV}$, pour un plasma d'argon. Il a vérifié la dépendance en $\sqrt{n}$, trouvant la valeur de $3,1 \Omega^{-1} \mathrm{~cm}^{-1}$ pour une densité électronique de $n_{\mathrm{e}} \simeq 10^{13} \mathrm{~cm}^{-3}$. En extrapolant cette valeur pour $n_{\mathrm{e}}=10^{19} \mathrm{~cm}^{-3}$, nous obtenons $3100 \Omega^{-1} \mathrm{~cm}^{-1}$ qui est encore trop élevé. Les mesures de Hamberger, faites à température constante, ne contiennent pas la loi de variation de la conductivité avec la température. Nous avons constaté dans notre décharge une dépendance en $T^{3 / 2}$. Cette dépendance diviserait la valeur calculée précédemment par 30 et nous obtiendrions $100 \Omega^{-1} \mathrm{~cm}^{-1}$ dans la gamme des valeurs que nous avons déterminées. 
Le mécanisme de la conduction fait donc intervenir des turbulences microscopiques. Mais il reste à mieux préciser les valeurs expérimentales, $\sigma, n_{\mathrm{e}}$ et $T$ pour faire une étude quantitative de ces instabilités.

5. Conclusion. - Les mesures optiques et électriques effectuées sur une décharge impulsive intense injectée dans un filament gazeux ionisé conduisent au modèle suivant de la structure de cette décharge :

Au temps $t: 300 \mathrm{~ns}$, le diamètre de la décharge est $2 \mathrm{~mm}$, la densité de courant moyenne est $1,7 \mathrm{ma} / \mathrm{cm}^{2}$. La pression dynamique est de quelques kbars et la pression magnétique de 1,6 kbar. La densité électronique moyenne dans la décharge est de l'ordre de $10^{19} \mathrm{~cm}^{-3}$, la température de $1 \mathrm{keV}$. Cette décharge est entourée d'une couche mince, de densité électronique de l'ordre de $10^{21} \mathrm{~cm}^{-3}$ de température $10 \mathrm{eV}$ et d'épaisseur $5 \mu \mathrm{m}$.

Ce plasma a été produit avec une énergie stockée relativement modeste de $8 \mathrm{~kJ}$. L'étude de ce type de plasma est intéressante car on peut $\mathrm{y}$ mesurer des coefficients de transport utiles pour les recherches liées à la fusion thermonucléaire par compression laser. Cette étude a permis de mettre en évidence l'effet d'une injection rapide de l'énergie dans le filament gazeux ionisé.

Remerciements. - Nous tenons à remercier le C.E.A. (Limeil) pour une aide importante en matériel scientifique et pour sa coopération.

Nous remercions M. Berthou de l'université Pierre et Marie Curie pour les mesures que nous avons pu effectuer avec son densitomètre.

Nous remercions $M$. Blanchet et le laboratoire Central de l'Armement pour leur collaboration technique.

Ce travail a bénéficié du concours de la R.C.P. 268 Plasmas à forte corrélation.

Ce travail a été effectué au sein de l'Équipe de Recherche 198 Plasmas Denses du C.N.R.S. associée à l'université Pierre et Marie Curie.

\section{Bibliographie}

[1] Brush S. G., Sahlin H. L., et Teller E., J. Chem: Phys. 45 (1966) 2102 Hansen J. P., Phys. Rev. A 8 (1973) 3096.

[2] Deutsch E., Lavaud M., Phys. Rev. A 9 (1974) 2598.

[3] Norman G. E., et Starostin A. N., High Temp. 8 (1970) 381. Klimontovitch J. L., Théorie cinétique des gaz et des plasmas non idéaux, Moscou 1975 (Pergamon Press). Ebeling W., KraefT W. D., KremP D., Theory of bound states and ionization equilibrium in Plasmas and solids. Ergeb. Plasmaphys. Gaselektronik bd 5 Akademic-Verlag Berlin (1976).

[4] Mather J. W., Conf. on Plasma Physics and controlled fusion research Culham. Sept. 1965.

Patou C., Simonnet A., Watteau J. P., J. de Physique 29 (1968) 973, Patou C., J. de Physique 31 (1970) 339.

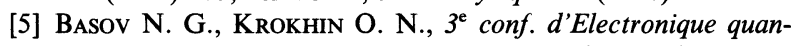
tique. Paris 1963, BoBIN J. L., B.I.S.T. du C.E.A. $\mathrm{n}^{\circ} 130$, octobre 1968. Bobin J. L., Floux F., Langer P., PigneROL H., Phys. Lett. 28 (1968) 298.

[6] Jablon C., Rioux C., Nucl. Fusion 16 (1976) 791.

[7] Exploding wires edited by W. G. Chace and H. K. Moore (Plenum Press) 1960-1962-1964-1968.

[8] Skowronek M., Rous J., Goldstein A., Cabannes F., Phys. Fluids 13 (1970) 378.

[9] Ogurtsova N. N., PodmoshenskiI I. V., Smirnov Y. L., High Temp. 14 (1976) 1.
[10] Allen J. E., Craggs J. D., Britt J., Appl. Phys. 5 (1954) 446. [11] Borovick E. S., Kantdesal V. P., Knyazer Yu. R., Mitin R. V., Petrenko V. I., Sov. Phys. Tech. Phys. 12 (1967) 502.

[12] SkowroneK M., C. R. Hebd. Séan. Acad. Sci. 250 (1960) 1808.

[13] Delmas M., Delobeau F., Jolas A., Watteau J. P., Revue Phys. Appl. 2 (1967) 7.

[14] Cabannes F., SkowroneK M., 8th I.C.P.I.G. Vienne 1967243.

[15] Weizel W., Rompe R., Theorie Elektrischer Lichtbögen und Funken (J. A. Barth, Leipzig), 1949.

[16] Vlastos A. E., J. Appl. Phys. 43 (1972) 1987.

[17] Popovic M. M., Popovic S. S., Gia V. T., Roméas P., SkowRONEK M., 4th int. Conf. On gas Discharges (Swansea) 1976, 125 .

[18] SPITZER L. Jr., Physics of Fully ionized Gases (2nd edition) (J. Wiley and Sons, New York) 128.

[19] Kapitza P. L., Sov. Phys. J.E.T.P. 30 (1970) 973. Commisso R. J., Griem H. R., Phys. Fluids 20 (1977) 49.

[20] Rosenbluth M. N., Dagazian R. Y., Rutherford P. H., Phys. Fluids 16 (1973) 1894.

[21] Buneman O., Phys. Rev. 115 (1959) 503.

[22] Dupree T. H., Phys. Rev. Lett. 25 (1970) 789.

[23] Hamberger S. M., Friedman M., Phys. Rev. Lett. 21 (1968) 674. 